\title{
Evaluation of the Interference of $\mathrm{HbE}$ on $\mathrm{HbA1c}$ Results on the Detection System Produced by Shenzhen Lifotronic
}

Yaozong Hu

Zhongshan Hospital of Sun Yat-Sen University

Minghuan Suo (D 545141974@qq.com )

Zhongshan Hospital of Sun Yat-Sen University

Yan Xu

Chongqing University

Wenyong $\mathrm{Hu}$

Shenzhen Lifotronic Technology Co .,Ltd

Ting Hu

Zhongshan Hospital of Sun Yat-Sen University

Jiamin Huang

Zhongshan Hospital of Sun Yat-Sen University

Weijia Wang

Zhongshan Hospital of Sun Yat-Sen University

Kang Chen

Zhongshan Hospital of Sun Yat-Sen University

\section{Research Article}

Keywords: Hemoglobin E, Hemoglobin A1c, Interference

Posted Date: October 11th, 2021

DOI: https://doi.org/10.21203/rs.3.rs-914953/v1

License: (c) (i) This work is licensed under a Creative Commons Attribution 4.0 International License. Read Full License 


\section{Abstract}

Objective: To analyze the interference of $\mathrm{HbE}$ on the $\mathrm{HbA} 1 \mathrm{c}$ results on detection systems manufactured by Shenzhen Lifotronic.

Methods: Volunteers were recruited from Zhongshan city, Guangdong province during June 2020 to November 2020. A total of 55 specimens were obtained, including 40 cases without $\mathrm{HbE}(23$ cases in normal group and 17 cases in diabetes group) and 15 cases with $\mathrm{HbE}$ variant. $\mathrm{HbA} 1 \mathrm{c}$ of these two groups (normal and $\mathrm{HbE}$ group) were analyzed by Shenzhen Lifotronic H8, H9 and GH-900Plus, Bio-Rad Variant II Turbo 2.0 and D-10 from USA, Arkray HA-8180 from Japan, statistical analysis was conducted on the difference between detection results of normal group and $\mathrm{HbE}$ variant group. Bio-Rad Variant II Turbo 2.0 was used as the comparison system to calculate the bias and to evaluate the interference of $\mathrm{HbE}$ on each system.

Results: In normal group, the results of each detection system were comparable to those of Variant II Turbo 2.0, with a bias of $-6 \% \sim 6 \%$. In $\mathrm{HbE}$ variant group,system bias of $\mathrm{H} 8, \mathrm{H} 9, \mathrm{GH}-900$ Plus and $\mathrm{D}-10$ was within $-6 \%$ and $6 \%$, and the results showed no significant difference $(P>0.05)$, while the negative bias of HA-8180 showed significant difference $(P<0.05)$.

Conclusion Compared with Bio-Rad Variant II Turbo 2.0, HbA1c can be correctly detected by H8, H9 and $\mathrm{GH}-900 \mathrm{Plus}$ system without the interference of $\mathrm{HbE}$.

\section{Introduction}

Hemoglobin A1c (Glycated hemoglobin, $\mathrm{HbA1c}$ ) is widely used in clinical practice as an important indicator to assess the average level of blood glucose control. China has paid increasingly attention to the standardization of $\mathrm{HbA} 1 \mathrm{c}$ test results, and the research and development of $\mathrm{HbA} 1 \mathrm{c}$ test system has been constantly innovating. It is particularly important to develop a detection system with good precision and strong anti-interference ability to accurately meet the needs of clinical detection ${ }^{1,2}$. $\mathrm{HbE}$ is one of the most common abnormal hemoglobin proteins in the world, with high incidence rate in China, India, Southeast Asia and North America ${ }^{3}$. Hemoglobin variation can interfere with $\mathrm{HbA} 1 \mathrm{c}$ test results to varying degrees ${ }^{4}$, the purpose of this study is to discuss the $\mathrm{HbA1c}$ testing results in the presence of $\mathrm{HbE}$ variant by Shenzhen Lifotronic's H8, H9 and GH-900Plus detection systems. The comparison between Shenzhen Lifotronic's three system and Bio-Rad Variant II Turbo 2.0 with regard to the interference degree of $\mathrm{HbE}$ was evaluated. The results are reported as follows.

\section{Materials And Methods}

\section{Source of specimens}


Volunteers from Zhongshan Hospital of Sun Yat-Sen University were recruited from June 2020 to November 2020. A total of 55 EDTA-K $\mathrm{K}_{2}$ anticoagulant venous blood samples were obtained in this study, including 40 cases in normal group,which were used as normal specimen for comparison experiment without hemoglobin variant, hyperlipidemia, uremia and other interfering factors. In addition, $15 \mathrm{HbE}$ variant samples were preliminarily determined $\mathrm{HbE}$ presence by capillary electrophoresis, and then genotype was detected and confirmed by gene sequencing. All the volunteers signed informed consent forms, and the study was reviewed by the Medical Ethics Committee of Zhongshan Hospital.

\section{Specimen collection}

Fasting blood of $2 \mathrm{~mL}$ EDTA- $\mathrm{K}_{2}$ anticoagulant venous blood was collected. These samples were used for capillary electrophoresis, gene sequencing and $\mathrm{HbA} 1 \mathrm{c}$ test. All collected specimens were pipetted and stored at $-70^{\circ} \mathrm{C}$.

\section{Detection system and method}

$\mathrm{HbA1c}$ was detected by lon Exchange High Performance Liquid Chromatography (IE-HPLC) using Shenzhen Lifotronic's automatic H8, $\mathrm{H} 9$ and $\mathrm{GH}-900 \mathrm{Plus} \mathrm{HbA} 1 \mathrm{c}$ analyzers and reagents, calibrators, control material and consumables.

$\mathrm{HbA1c}$ was detected by lon Exchange High Performance Liquid Chromatography using Variant II Turbo 2.0, D-10 automatic HbA1c analyzers and reagents, calibrators, control material and consumables (BioRad Company).

HbA1c was detected by lon Exchange High Performance Liquid Chromatography using Arkray HA-8180 automatic $\mathrm{HbA} 1 \mathrm{c}$ analyzer and reagents, calibrators, control material and consumable.

\section{Sample Detection}

55 specimens were tested by H8, H9, GH-900Plus, Variant II Turbo 2.0, D-10, and HA-8180 respectively. The NGSP quality evaluation material was retested at Variant II Turbo 2.0 before experimental operation to ensure that the system met the requirements of NGSP laboratory certification, so as to ensure the traceability of the comparison system. The relative bias between detection results of different systems and Variant II Turbo 2.0 was calculated to be within $-6 \% \sim+6 \%$. Meeting this condition means that detection results of other systems are comparable to those of Variant II Turbo 2.0.

\section{Statistical analysis}

$\mathrm{HbA} 1 \mathrm{c}$ tests were respectively performed on the experimental systems and the comparison system for each group. Statistical analysis was carried out for the bias of detection results. All the data were statistically analyzed by Excel and SPSS 22.0 software. Kolmogorov-Smirnov test, a single sample, was used to test the normality of the measurement data, which were in line with the normal distribution, and paired sample t-test was used as well. $P<0.05$ was considered statistically significant. 


\section{Results}

\section{Retesting results of NGSP in Variant II Turbo 2.0 system}

All the NGSP quality assessment and target value bias detected on the same day were less than $6 \%$, detailed results are shown in Table 1. Indicating that the accuracy of the analysis and testing system reaches acceptable standards to ensure that the system meets the requirements of NGSP laboratory certification, so as to ensure the traceability of the comparison system.

Table 1

Retesting results of NGSP quality evaluation in Variant II Turbo 2.0 system

\begin{tabular}{|llllllll|}
\hline Serial No. & NGSP target value & Value 1 & Bias \% & Value 2 & Bias \% & Value 3 & Bias \% \\
\hline 1 & 5.54 & 5.6 & 1.08 & 5.6 & 1.08 & 5.5 & -0.72 \\
\hline 2 & 6.31 & 6.2 & -1.74 & 6.3 & -0.16 & 6.3 & -0.16 \\
3 & 9.64 & 9.5 & -1.45 & 9.6 & -0.41 & 9.6 & -0.41 \\
\hline
\end{tabular}

\section{Comparison and bias analysis of HbA1c test results of the five detection systems}

The correlation coefficient $r$ of regression analysis between the five detection systems including $\mathrm{H} 9, \mathrm{H} 8$, GH-900Plus, D-10 and HA-8180 and the comparison system of Variant II Turbo 2.0 were all greater than 0.975 , and the percentage bias of the experimental system and the comparison system were all less than $\pm 6 \%$, the specific results were shown in Table 2 .

Table 2

Comparison of $\mathrm{HbA} 1 \mathrm{c}$ results between the five detection systems and comparison system

\begin{tabular}{|llll|}
\hline $\begin{array}{l}\text { The experimental } \\
\text { system }\end{array}$ & $\begin{array}{l}\text { The regression } \\
\text { equation }\end{array}$ & $\begin{array}{l}\text { The correlation } \\
\text { coefficient }\end{array}$ & $\begin{array}{l}\text { Normal group bias } \\
\text { range \% }\end{array}$ \\
\hline H9 & $Y=0.967 x+0.235$ & 0.997 & $-2.9 \sim 5.7$ \\
\hline H8 & $Y=0.961 x+0.293$ & 0.997 & $-2.6 \sim 3.8$ \\
\hline GH-900Plus & $Y=0.967 x+0.235$ & 0.998 & $-1.7 \sim 5.9$ \\
\hline$D-10$ & $Y=0.966 x+0.184$ & 0.997 & $-5.2 \sim 3.5$ \\
\hline HA-8180 & $Y=0.957 x+0.307$ & 0.995 & $-3.8 \sim 5.7$ \\
\hline
\end{tabular}




\section{Chromatogram analysis of $\mathrm{HbE}$ variant in different $\mathrm{HbA1c}$ detection systems}

The elution time of $\mathrm{H} 9$ was 360 seconds, and the peak names were HbA1a, HbA1b, HbF, LA1c, HbA1c, P3, $\mathrm{P} 4, \mathrm{HbAO}, \mathrm{HbA} 2$ and $\mathrm{HbE} / \mathrm{D} / \mathrm{S} / \mathrm{C}$ (if any) respectively. The average retention time of $\mathrm{HbE}$ was 220 seconds, which is after $\mathrm{HbA} 2$. Moreover, it was identified and reported separately in the chromatogram, and the system could directly indicate suspected $\mathrm{HbE}$ variant.

The elution time of $\mathrm{H} 8$ and $\mathrm{GH}-900 \mathrm{Plus}$ was 130 seconds, and the peak names were $\mathrm{HbA} 1 \mathrm{a}, \mathrm{HbA} 1 \mathrm{~b}, \mathrm{HbF}$, $\mathrm{LA} 1 \mathrm{c}, \mathrm{HbA1c}, \mathrm{HbAO}$ and variant window respectively. The average retention time of $\mathrm{HbE}$ was 89 seconds which is after $\mathrm{HbAO}$ in the chromatogram, and the system gave V-window alarm.

The elution time of Variant II Turbo 2.0 was 90 seconds, and the peak names were $\mathrm{HbA1a}, \mathrm{HbA} 1 \mathrm{~b}, \mathrm{HbF}$, $\mathrm{LA1C}, \mathrm{HbA1c}, \mathrm{P} 3, \mathrm{P} 4$ and $\mathrm{HbA0}$ respectively. The average retention time of the variant was 66 seconds, $\mathrm{HbE}$ 's retention time is after $\mathrm{HbAO}$, and it was next to $\mathrm{HbAO}$, but the obvious presence of this variant could be identified from the chromatogram, and the system gave $\mathrm{V}$-window alarm too.

The elution time of D-10 was 180 seconds, and the peak names were approximately $\mathrm{HbA} 1 \mathrm{a}, \mathrm{HbA} 1 \mathrm{~b}, \mathrm{HbF}$, $\mathrm{LA1c}, \mathrm{HbA1c}, \mathrm{P} 3$, and $\mathrm{HbA0}$, respectively. The average retention time of $\mathrm{HbE}$ variant was 95 seconds, which is after $\mathrm{HbAO}$, and it was next to $\mathrm{HbAO}$, but the obvious presence of this variant could be identified from the chromatogram, and the system gave $\mathrm{V}$-window alarm too.

The elution time of HA-8180 was 48 seconds, and the peak names were approximately HbF, LA1c, HbA1c, and $\mathrm{HbAO}$, respectively. The retention time of $\mathrm{HbE}$ was between $\mathrm{HbA} 1 \mathrm{c}$ and $\mathrm{HbAO}$, and it was after $\mathrm{HbA} 1 \mathrm{c}$ in the chromatogram. The system showed no variant alarm or there was no peak name or retention time.

\section{Statistical analysis of $\mathrm{HbA1c}$ test results differences between normal group and $\mathrm{HbE}$ variant group.}

There was no significant difference between $\mathrm{HbA} 1 \mathrm{c}$ and $\mathrm{HbE}$ group detected by $\mathrm{H} 8, \mathrm{H} 9, \mathrm{GH}-900$ plus, Turbo 2.0 and D-10 ( $>0.05)$, while there was significant difference between $\mathrm{HbA} 1 \mathrm{C}$ and $\mathrm{HbE}$ group detected by HA-8180 $(P<0.05)$, as shown in Table 3 . 
Table 3

$\mathrm{HbA} 1 \mathrm{c}$ test results of normal group and $\mathrm{HbE}$ group (\%)

\begin{tabular}{|lll|}
\hline The experimental system & $\begin{array}{l}\text { normal group } \\
\text { HbA1c } \%\end{array}$ & HbE variant group HbA1c\% \\
\hline H9 & $5.69+/-0.38$ & $5.48+/-0.52$ \\
\hline H8 & $5.66+/-0.43$ & $5.55+/-0.55$ \\
\hline GH-900Plus & $5.75+/-0.41$ & $5.65+/-0.56$ \\
\hline Turbo 2.0 & $5.60+/-0.42$ & $5.50+/-0.53$ \\
\hline D-10 & $5.60+/-0.42$ & $5.48+/-0.47$ \\
\hline HA-8180 & $5.65+/-0.45$ & $4.44+/-0.28 \mathbf{4}$ \\
\hline Note: $\Delta$ Comparison of HbA1c results between normal group and HbE group $(P<0.05)$ \\
\hline
\end{tabular}

\section{Bias analysis of $\mathrm{HbE}$ variants in different $\mathrm{HbA1c}$ detection systems}

D-10, H9, H8 and GH-900plus showed no significant difference ( $P>0.05)$ with the compared system( bias $< \pm 6 \%$, while HA-8180 showed significant difference $(P<0.05)$ with negative bias $(P<0.05)$. As shown in Table 4 and figure 2 for details.

Table 4

Average bias of each experimental system and comparison system (\%)

\begin{tabular}{|lll|}
\hline The experimental system & Average bias \% of HbE-free group & Average bias \% of $\mathrm{HbE}$ group \\
\hline $\mathrm{H} 9$ & $0.45+/-2.42$ & $0.34+/-1.99$ \\
\hline H8 & $0.91+/-1.63$ & $0.94+/-1.58$ \\
\hline GH-900Plus & $2.34+/-2.07$ & $2.66+/-2.44$ \\
\hline D-10 & $0.47+/-2.05$ & $0.25+/-1.59$ \\
HA-8180 & $0.52+/-2.88$ & $18.95+/-4.25$ \\
\hline $\begin{array}{l}\text { Note: } \mathbf{\Delta} \text { Comparison of HbA1c results with comparison of system bias in HbE-free group and } \mathrm{HbE} \\
\text { variant group, P<0.05 }\end{array}$
\end{tabular}

\section{Discussion}

Methods for the detection of HbA1c include enzymatic method, immunoturbidimetry, affinity chromatography and ion-exchange liquid chromatography, among which the results of ion-exchange 
liquid chromatography (IE-HPLC) are more accurate with good repeatability and reproducibility. American Association of Clinical Chemistry (AACC) and International Federation of Clinical Chemistry (IFCC) suggest to use IE-HPLC as the one of the gold standards for the detection of HbA1c. Therefore, HPLC method is widely used in clinical practice. However, different detection systems based on HPLC method will report different $\mathrm{HbA1c}$ results with presence of variant due to the differences in column quality, reagent formulation and elution time, etc. ${ }^{5}$.

Professor Wen Dongmei and Xu Anping's research results show ${ }^{6,7}$ that the Variant II Turbo 2.0 has long testing time with high resolution. In addition, the elution time was 90 seconds, thus $\mathrm{HbE}$ variant peak can be well separated. Therefore, the system is not intefered by $\mathrm{HbE}$ variants and the results are consistent with NGSP web site. Moreover, testing lab for this experiment was accredited by NGSP as grade I laboratory, results accuracy is guaranteed and acceptable by different requirement. Therefore, Variant II Turbo 2.0 was chosen as the comparison of the experimental system to evaluate the $\mathrm{HbE}$ antiinterference ability.

These three HbA1c detection systems from Shenzhen Lifotronic have anti-interference ability to $\mathrm{HbE}$ variants. This study also shows that none of the $\mathrm{HbE}$ variants have any influence on the results of these three detection systems. $\mathrm{H} 9$ system variant mode was 360 seconds(graph is 300 seconds only) and $\mathrm{H} 8 / \mathrm{GH}-900 \mathrm{Plus}$ is 130 seconds. The chromatograms showed that $\mathrm{HbE}$ could be recognized by the detection systems and could be well separated. Among all the detection system used in this experiment, $\mathrm{H} 9, \mathrm{H} 8, \mathrm{GH}-900 \mathrm{Plus}$ and Variant II Turbo 2.0 system had the same degree of HbE variant recognition, indicating the presence in the Variant-Window. $\mathrm{H} 9$ variant mode can fully separate $\mathrm{HbE}$ variant, and the retention time of $\mathrm{HbE}$ is around 220s, which is about 0.73 quantile of the total graphic time.This parameter for $\mathrm{H} 8$ is $0.68, \mathrm{GH}-900$ Plus is 0.68 , and Variant II Turbo 2.0 is 0.73 . Retention time of $\mathrm{HbE}$ in these three Lifotronic systems is highly similar to Variant II Turbo 2.0.0ther studies have shown that ${ }^{8}$ the $\mathrm{H} 9$ system, although has a relatively low resolution of $\mathrm{Hb}$ NewYork, could indicate the presence of the variant through the chromatogram. The variant mode of $\mathrm{H} 9$ could identify 12 hemoglobin variants and preliminarily determine the type of the variant based on the retention time and peak shape of the variant. Due to relatively large number tests that can be supported by chromatographic column and reagent of $\mathrm{H} 9$ system, it reduces the frequency for replacement of chromatographic column and making calibration/QC in laboratory.It also helps the lab to save costs and ensures the stability of test results, so it is more suitable for large laboratories.

Compared with Variant II Turbo 2.0, D-10 system manufactured by Bio-Rad from USA has a longer elution time of 180 seconds, and the $\mathrm{HbE}$ Variant can be completely separated. Therefore, the anti-interference ability of $\mathrm{HbE}$ is consistent with that of Variant II Turbo 2.0 manufactured by the same company. However, its elution time is longer, and the number of tests can be supported by chromatographic column and reagent is less, so D-10 is more commonly used in small laboratories.

The Arkray HA-8180 from Japan has the feature of fast speed.. Its elution time is 48 seconds, which is the fastest in the detection systems of this study. Its fast mode is often used for mass screening. 
However, the elution time is too short to separate the $\mathrm{HbE}$ peak which leads to relatively large negative bias in the detection results. Therefore, $\mathrm{HA}-8180$ was not suitable for the detection of $\mathrm{HbA} 1 \mathrm{c}$ results for population with $\mathrm{HbE}$ variants.

In conclusion, $\mathrm{H} 8, \mathrm{H} 9$ and $\mathrm{GH}-900 \mathrm{Plus} \mathrm{HbA} 1 \mathrm{c}$ detection systems and Bio-Rad Variant II Turbo 2.0 have comparable results when testing $\mathrm{HbA} 1 \mathrm{c}$ in population with $\mathrm{HbE}$ variants. And the anti-interference ability

of these three detection systems from Shenzhen Lifotronic was better than that of Arkray's HA-8180. With continuous improvement of reagent formula and chromatographic column quality, HbA1c detection systems from Shenzhen Lifotronic have strengthened their ability to separate and identify the variants and have a good performance on anti-interference of variants. In regions where hemoglobin variants appear frequently, it is necessary to fully understand the anti-interference ability of each detection system against the variants, so as to avoid results bias of $\mathrm{HbA1c}$, which would lead to misjudgment of average blood glucose when monitoring level of patients by clinicians.

\section{Declarations}

\section{ACKNOWLEDGMENTS}

Thanks to all the volunteers from Zhongshan.

\section{Ethical approval:}

Informed consent was obtained from all individual participants. This study protocol conforms to ethical guidelines of World Medical Association. The Medical Ethics Committee of Zhongshan Hospital reviewed this protocol, and all volunteers gave their informed consent for the inclusion in this study.

\section{Approval of the research protocol:}

This study was approved by Zhongshan Science and Technology Bureau.

\section{Informed Consent:}

Informed consent was obtained from all individual participants included in the study.

Approval date of Registry and the Registration No: Zhongshan Science and Technology Bureau approved this study on January 2018.( Registration No. 2018B1036)

\section{Animal Studies:}

$\mathrm{N} / \mathrm{A}$ 


\section{Conflict of interest:}

The authors declare no conflict of interest.

Data Policy:Some or all data, models, or code generated or used during the study are available from the corresponding author by request.

\section{Authors' Contributions:}

$\mathrm{Hu} \mathrm{YZ}$ and Suo MH are responsible for the design of experimental scheme, the collection of samples and the writing of articles.

$\mathrm{Hu} \mathrm{YZ}$ and Suo MH contributed equally to this work.

$\mathrm{Xu} \mathrm{Y}$ and Hu WY are responsible for the arrangement of instrument detection principle, time and advantages.

$\mathrm{Hu} \mathrm{T}$ and Huang $\mathrm{JM}$ are responsible for searching the experimental population, contacting the volunteers by telephone, and guiding the volunteers to collect samples. Wang WJ and Chen $\mathrm{K}$ responsible for the collection, detection and preservation of specimens.

\section{References}

1. Sacks DB, Bruns DE, Goldstein DE, et al. Guidelines and Recommendations for Laboratory Analysis in the Diagnosis and Management of Diabetes Mellitus. Clinical Chemistry 2002;48(3):436-472.

2. Zhang CB, Zhang TJ. Face the new challenges in the standardization of hemoglobin A1C measurements in China, Chin J Lab Med 2018;41(011):797-799.

3. Weatherall DJ, Clegg JB. Inherited haemoglobin disorders: an increasing global health problem. Bulletin of the World Health Organization 2001; 79(8):704.

4. Thom CS, Dickson CF, Gell DA, et al. Hemoglobin Variants: Biochemical Properties and Clinical Correlates. Cold Spring Harbor Perspectives in Medicine 2013;3(3):306-307.

5. Curt R, Shawn C, Steven $\mathrm{H}$, et al. Validation of the Use of Trinity Biotech ultra2 as a Comparative Method for Hemoglobin A1c Measurements in the Presence of $\mathrm{HbE}$ and $\mathrm{HbD}$-Punjab Traits. Clinical Chemistry 2016;63 (2):608-610.

6. Wen DM, Zhang XM, Zhang DC, et al. Interference of hemoglobin E on measurements of glycosylated hemoglobin (HbA1c), Chin J Lab Med 2014, 37(012):921-927.

7. Xu AP, Xia Y, Ji L, et al. Influences of hemoglobin variants on different HbA1c measurement systems, Chin J Lab Med 2015, 38(7):470-474.

8. Li R, Tang H, Kan L, et al. Evaluation on the separated effect of 13 hemoglobin variants by a new automatic HbA1c analyzer, J Clin Lab Anal 2020,34(10): e23446 
Figures

H9
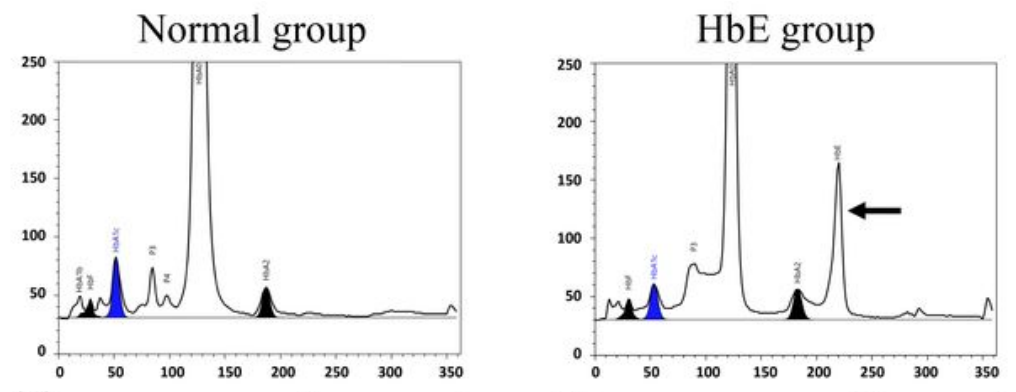

H8
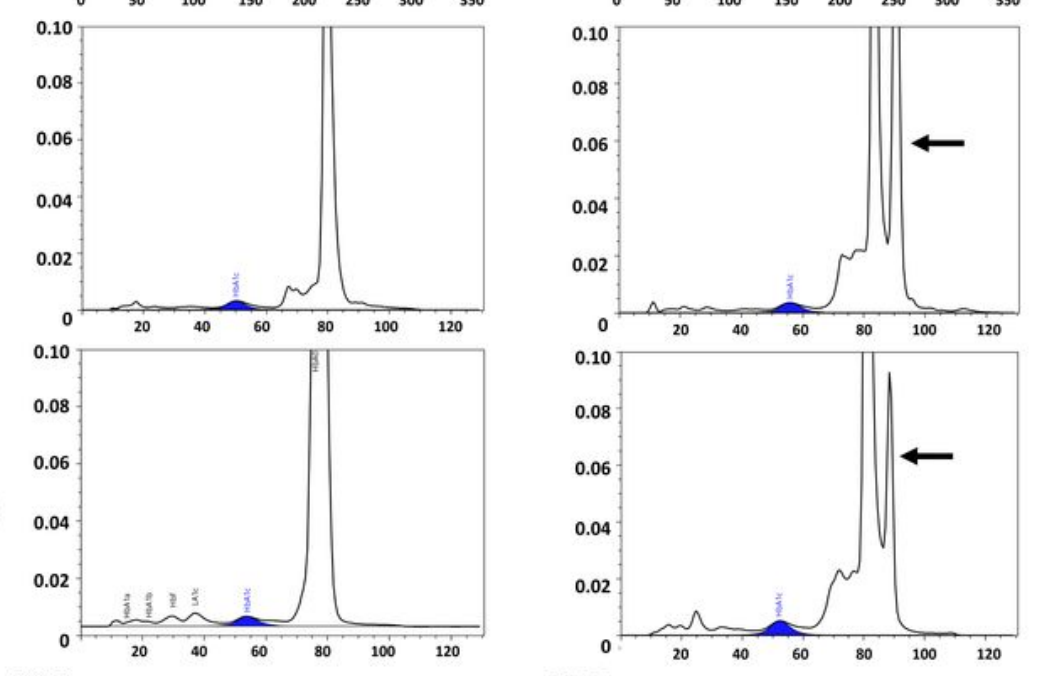

GH-900Plus
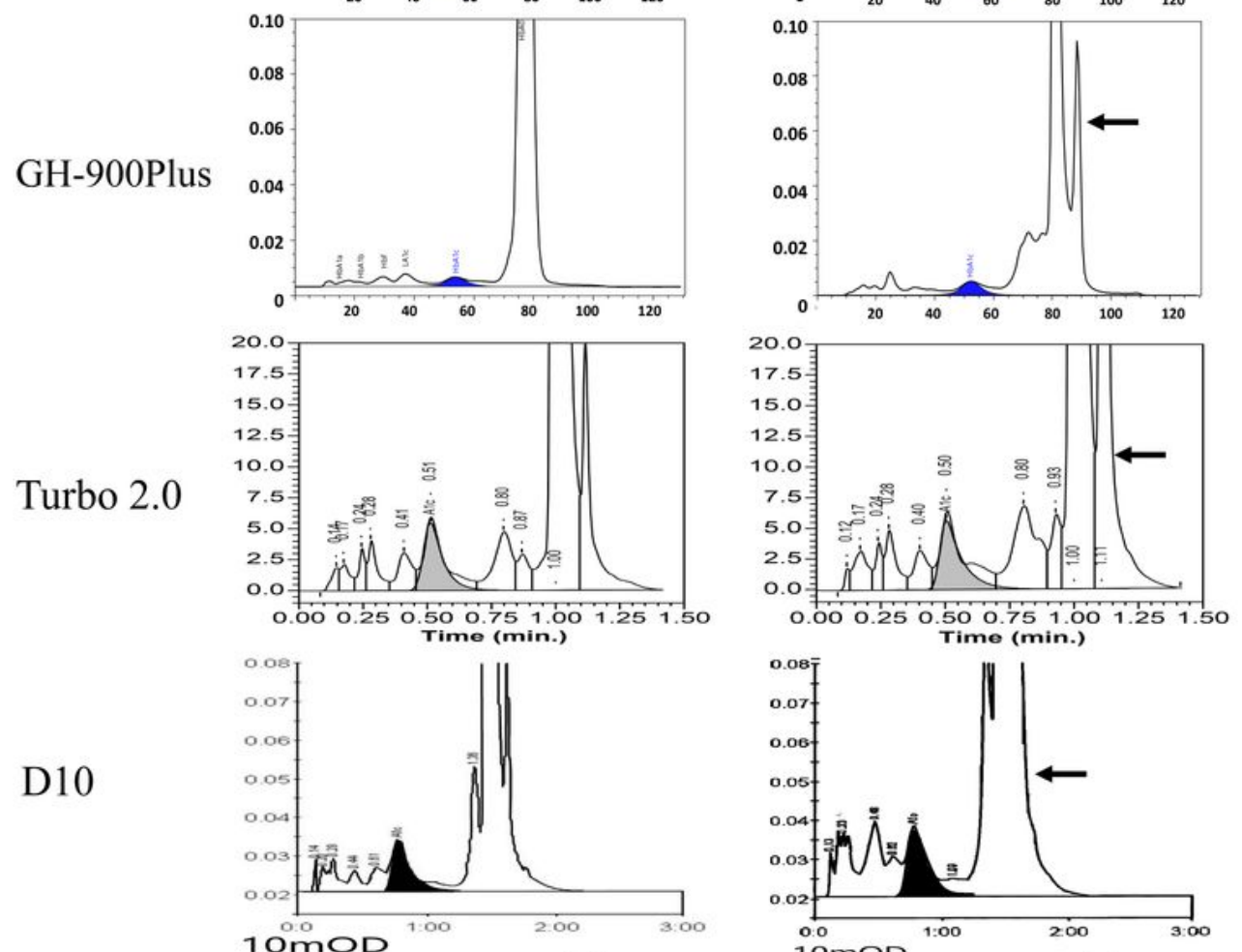

D10
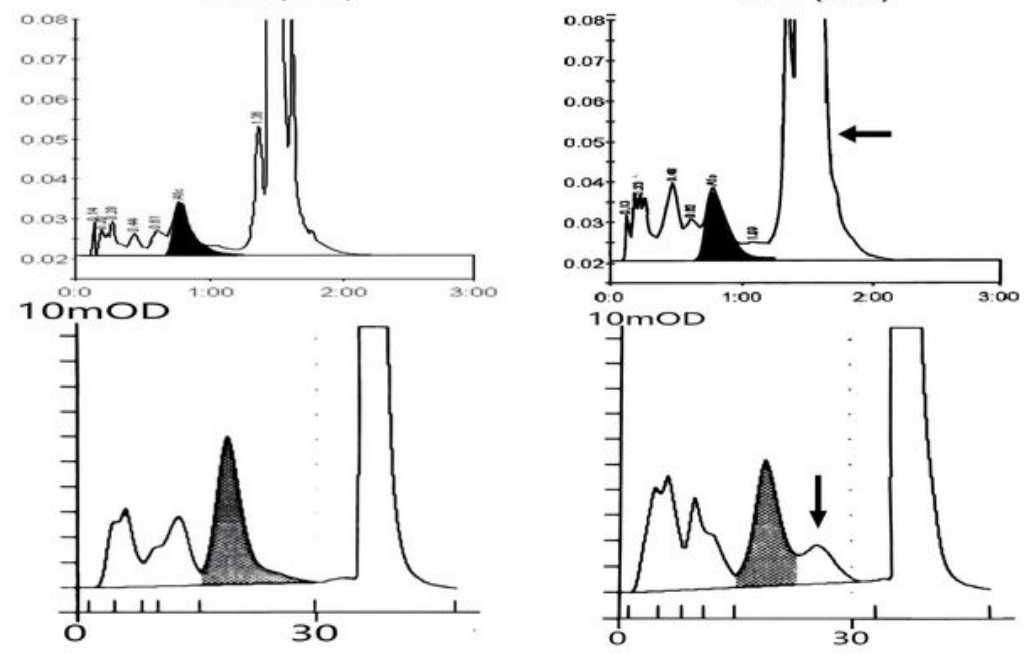

Figure 1

Chromatograms of normal and $\mathrm{HbE}$ group in 6 detection systems $\leftarrow$ - Represents the HbE position in Chromatogram 


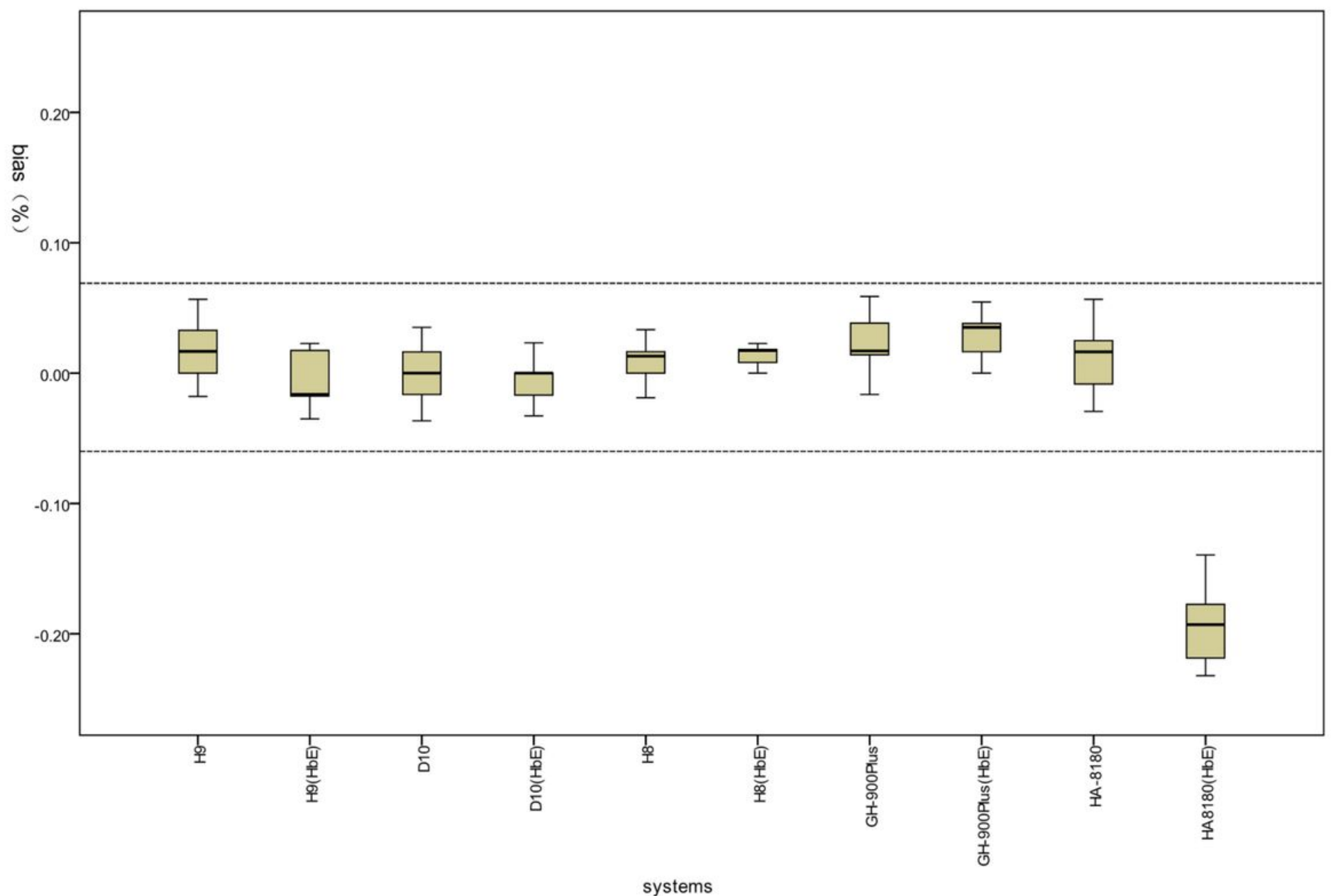

Figure 2

5 Bias distribution of $\mathrm{HbA1c}$ detection results in 5 detection systems $\mathrm{X}$-axisrepresents different systems $\mathrm{Y}$-axis represents bias between test results and comparativemethod(\%) Dottedlines representbias within $-6 \% \sim+6 \%$. 